\title{
¿CONDUCE LA NEUROCIENCIA \\ A UNA NATURALIZACIÓN DEL DEONTOLOGISMO? «JUICIOS MORALES DEONTOLÓGICOS» EN PERSPECTIVA NEUROÉTICA
}

\author{
JAVIER GRACIA CALANDÍN \\ Universidad de Valencia
}

\begin{abstract}
RESUMEN. A tenor de las investigaciones en el campo de la neuroética y más en concreto en la teoría del proceso dual del juicio moral en autores como Joshua Greene, nos planteamos la cuestión de si la neurociencia conduce necesariamente a una «naturalización del deontologismo». De este modo en nuestra comunicación ponemos en cuestión un modo de hacer neurociencia que pretenda reducir el deontologismo a un tipo de respuesta exclusivamente emocional. Frente a este modelo planteamos otra forma de hacer «neuroética» donde se consideren las estructuras psicofísicas de la moralidad sin incurrir en el reduccionismo miope naturalista. Se trata, por lo tanto, de descubrir la neurociencia de la ética, esto es, la naturaleza de la moralidad sin que eso implique reducir la moralidad a la naturaleza. Así entendida la neurociencia puede contribuir al trabajo interdisciplinar de comprender la vasta naturaleza humana en toda su amplitud.
\end{abstract}

PALABRAS CLAVE: neuroética, naturalización, juicios morales deontológicos, Joshua Greene.

\section{Does Neuroscience lead to a Naturalization of Deontologism? Reflection on the "Deontological Moral Judgments" from a neuroethical approach}

ABSTRACT. According to the research in the field of neuroethics and more specifically in the theory of dual process of moral judgment, in authors such as Joshua Greene, we pose the question whether neuroscience leads ineluctably to a «naturalization of Deontologism». So, in my communication I criticize a way of doing neuroscience by reducing deontological moral judgments exclusively to a kind of emotional response. Beyond this model I propose another way of doing «neuroethics» where the psychophysical structures of morality are considered without incurring the myopic reductionist naturalism. According to this second approach, it is about discovering the neuroscience of ethics, namely, the nature of morality without reducing morality to nature. From that approach, neuroscience can contribute to an interdisciplinary work in order to understand the vast human nature in all its breadth.

KEY WORDS: neuroethics, naturalization, deontological moral judgment, joshua greene.

1. CRíticas a las pretensiones de «nATURALIZAR El DEONTOLOGismo» POR PARTE DE JoSHuA GREENE

Recientemente, a tenor de los estudios experimentales en neurociencia autores como Baron, Haidt, Greene, Small, Loewenstein, Quervain, Sanfey, Carlsmith, entre otros, han afirmado que en nuestro cerebro se pueden distinguir dos tipos de

<?> Proyecto de Investigación Científica y Desarrollo Tecnológico FFI2013-47136-C2-1-P, financiado por el Ministerio de Economía y Competitividad con Fondos FEDER de la Unión Europea. 
procesos cognitivos en la toma de decisiones morales. Por una parte habría un tipo de juicios morales que implican procesos cognitivos vinculados con las emociones y asociados con actividad localizada en la amígdala, la corteza prefrontal media y corteza prefrontal ventromedial. Por otra parte, habría otro tipo de juicios morales que implican procesos cognitivos vinculados con el cálculo consciente y controlado. Es a esto a lo que se conoce como la «Teoría del proceso dual del juicio moral».

Lo novedoso de estos estudios es que consideran que son los «juicios morales característicamente deontológicos» los «inconscientes, irreflexivos e intuitivos». Así, en contra de lo que desde la filosofía moral se ha pensado tradicionalmente (al menos, desde Kant), no es la actividad racional consciente y argumentativa la que conduciría a juicios morales deontológicos, sino las intuiciones inconscientes y automáticas ${ }^{1}$. De este modo, la psicología cognitiva es presentada como la disciplina capaz de desvelar el «alma oculta de la filosofía moral kantiana» ${ }^{2}$.

Lo que desde este planteamiento se pretende no es solo descubrir las bases neuronales de los juicios morales deontológicos, lo cual sería legítimo aunque ciertamente difícil de determinar (como señalaré en la segunda parte de este trabajo, creo que las neurociencias pueden descubrir un mundo enormemente valioso para la ética, que nos permiten comprender más y mejor los mecanismos cerebrales que intervienen en la toma de decisiones). Lo que se pretender es naturalizar el deontologismo de modo que la única y verdadera razón de ser de éste sea desvelada por la neurociencia. Tal y como concluye Greene para explicar qué son los juicios morales deontológicos habría que acabar por prescindir de la filosofía moral deontológica, que al fin y al cabo se basa en un malentendido.

Por mi parte, yo no tendría inconveniente en reconocer que los juicios morales deontológicos se basan en elementos intuitivos o emocionales. Incluso el propio Kant se habría referido a las "prenociones estéticas de la receptividad del ánimo para los conceptos del deber en general» ${ }^{3}$. para llamar la atención de la necesidad de «sentimientos» que mueven a la razón pura práctica. Sin embargo, considero que es excesiva la pretensión de reducir los juicios morales deontológicos a respuestas emocionales automáticas, desacreditando las explicaciones filosóficas que buscan justificar normativamente el fenómeno moral. Vaciar de racionalidad los juicios morales deontológicos, tal vez sea pertinente para un planteamiento psicológico, pero en ningún caso incide en la cuestión normativa de fondo del deontologismo. De hecho el planteamiento de Greene adolece de un criterio claro para determinar cuándo es más adecuado confiar en una respuesta automática y cuándo conviene emplear el sistema manual para elaborar una respuesta más calculada ${ }^{4}$.

1 Vid. HAIDT, J., «El perro emocional y su cola racional. Un enfoque intuicionista social sobre el juicio moral», en Cortina, A. (ed.), Guía Comares de Neurofilosofía Práctica, Comares, Granada, 2012.

2 Greene, J., «The Scret Joke of Kant's Soul», en: Sinnot-Armstrong, W., Moral Psychology 3, The Neuroscience of Morality. Emotion, Brain Disorders, and Development, MIT Press, Cambridge, 2008, pp. 36-79.

3 Kant, I., La metafísica de las costumbres, Tecnos, Madrid, 1989, pp. 253-258.

4 Aunque él lo niegue, su confianza en que la ciencia resuelva los problemas normativos de la moral resulta excesiva: "If we believe that we ought to rely on automatic settings vs. manual mode to different extents in different situations, and if cognitive science can tell us when we are relying on automatic settings vs. manual mode, then cognitive science gives us normatively significant 
A mi juicio, Greene confunde el término «deontológico» y creo que se equivoca al afirmar que el racionalismo moral kantiano no busca emplear el razonamiento para determinar qué es lo correcto o válido, no pasando de ser el intento por justificar intuiciones preexistentes del hombre medio (intuition chasing process ${ }^{5}$ ). Creo que constituye una petición de principio (injustificada) pensar que hay una coincidencia entre las emociones automáticas y el deber prescrito por el imperativo categórico (Timmons, 2008: 97-99). ¿Realmente el imperativo categórico busca conservar las normas o convenciones establecidas? Los problemas se agravan cuando Greene recurre al consecuencialismo o "pragmatismo profundo" (Greene, 2013). Sin entrar directamente en los confusos e inconsistentes argumentos de Greene en su obra Moral Tribes (Greene, 2013) en favor del «pragmatismo profundo» (Wielenberg, 2014), tengo mis reservas para admitir que la ciencia sea la abanderada para contribuir al progreso moral. ¿Realmente la ciencia avanzará tanto para poder determinar qué respuestas son buenas o malas moralmente? ${ }^{6}$ ¿No se acaba incurriendo en la célebre falacia naturalista al pretender determinar desde la explicación neurocientífica qué es moralmente adecuado y qué no lo es?

Por mi parte creo que la solución no es naturalizar el deontologismo en una especie de «extensión natural de la práctica corriente y consistente con los compromisos históricos de la filosofía hacia el empirismo activo» (Greene, 2014: 726). Más bien creo que hay otra posible lectura de la importancia que la neurociencia tiene para le ética, a saber, no reducir la moralidad a naturaleza sino descubrir la naturaleza propia de la moralidad.

\section{DESCUBRIR LA NATURALEZA DE LA MORALIDAD SIN REDUCIR LA MORALIDAD A NATURALEZA}

En la segunda parte de este trabajo quiero esbozar de qué modo creo que la neurociencia puede contribuir a descubrir la naturaleza de la moralidad, incidiendo en la naturaleza que subyace y late en la moralidad, sin reducir la moralidad a naturaleza. Para ello es necesario plantear adecuadamente la interdisciplinariedad del tema, evitando caer en el problema de la anfibología terminológica. Tomar conciencia del carácter "híbrido» de los conceptos neuroéticos ha de conducir a aceptar que diferentes enfoques puedan contribuir a esclarecer la naturaleza de lo moral. A mi juicio, no basta con el método de las ciencias experimentales, sino que a su vez es preciso replantear las acepciones de racionalidad, emoción, conciencia, juicio moral, intuición, entre otras, que entran en juego, y hay que hacerlo desde planteamientos ético-filosóficos que no desatiendan el método de las ciencias humanas.

information -information that can nudges us, if not propel us, toward new and interesting normative conclusions». Greene, J., «Beyond Point-and-Shoot Morality: Why Cognitive (Neuro)Science Matters for Ethics», en: Ethics 124 (4), July, 2014, p. 715.

5 «One discerns, intuitively, the presence of rights and duties in particular cases, and then one searches for principles that might explain why those rights and duties are indeed present. I call this process intuition chasing», Ibid., 718.

6 "We need to understand the structure, origins, and limitations of our intuitive moral thinking, the better to know when our moral instincts are on target, and when they are giving us the right answers to the wrong questions». Ibid., 726. 
Por cuestión de espacio señalaré algunos aspectos de cómo ha de ser entendida la naturaleza de la moralidad en perspectiva neuroética.

En primer lugar, creo que es necesario distinguir entre una acepción descriptiva de moralidad y otra de tipo normativo ${ }^{7}$ para no incurrir, bien en la falacia naturalista (que pasa de una a otra), bien en el reduccionismo naturalista (que elimina el carácter normativo). Por ello me parece que es muy acertada la distinción entre «bases neuronales» $\mathrm{y}$ "fundamento moral» de los juicios morales ${ }^{8}$. Los enfoques de la neurociencia y de la biología evolutiva pueden explicar de modo más o menos solvente de qué manera el ser humano ha devenido un agente moral, cuáles son las capacidades que le definen filogenéticamente como tal. Cosa muy distinta sería pretender justificar la conducta humana en función del rendimiento evolutivo de ésta, la moral no queda subsumida en la naturaleza biológicamente entendida ${ }^{9}$.

Por eso resulta tan importante distinguir la capacidad para la ética de los sistemas o códigos de normas éticas ${ }^{10}$. Es razonable pensar que la capacidad para la ética pueda explicarse por la naturaleza biológica de los seres humanos. Pero cuando esta explicación alcanza a los códigos de normas éticas aceptados pretendiendo que éstos vengan determinados biológicamente es cuando se está optando por un planteamiento particular que es reduccionista y que se conoce como «naturalismo». A mi juicio podemos afirmar que la conducta moral se basa en mecanismos neuronales, sin por ello renunciar a criterios que no proceden del mecanismo evolutivo. El «deber moral» trasciende el mecanismo adaptativo de la evolución. Ello no implica entender la moral como algo «sobrenatural», pero sí como una naturaleza que no encuentra su validez necesariamente en los mecanismos adaptativos de la evolución, sino en códigos racionalmente (y no cerebralmente) fundamentados.

Por todo ello, a mi juicio, la naturaleza latente de la moralidad exige pensar con mayor radicalidad el vínculo que existe entre la racionalidad y la dimensión sentiente. Desde luego que los estudios neurocientíficos del juicio moral nos ayudan a entender mejor por qué nos afectan de forma diferente los problemas personales y los problemas impersonales. Pero conocer esta tendencia natural del cerebro humano no implica que debamos comportarnos de dicha manera. Este es el quicio en el que el «deontologismo» (bien entendido, esto es, no naturalizado) abre nuevos interrogantes, ¿debemos y queremos que nuestros juicios morales respondan exclusivamente al factor de proximidad o hay otros aspectos valiosos que han de determinarlo? ¿Es posible pensar en el cultivo de razón y emoción en un sentido que se elija el camino moralmente más valioso?

\footnotetext{
7 GERT, B.: «The definition of morality», en: ZaLTA, E.N. (comp.), Stanford Encyclopedia of Philosophy, http://plato.stanford.edu/entries/morality-definition (consultado el 1 de marzo de 2016).

8 Cortina, A., Neuroética y neuropolítica. Sugerencias para la educación moral, Tecnos, Madrid, 2011, pp. 77-96.

9 Teruel, P. J., «En torno a la posibilidad de naturalizar la ética», en: Flamarique, L. (ed.), Las raíces de la ética y el diálogo interdisciplinar, Biblioteca Nueva, Madrid, 2012 pp. 207-222.

10 Ayala, F., La evolución de un evolucionista, Valencia, PUV, Valencia, 2006, 352ss.
} 


\section{LA PROPIA AUTORÍA COMO PRESUPUESTO DE UNA RACIONALIDAD IN-CORPORADA}

Una buena articulación de racionalidad y corporalidad constituye uno de los principales desafíos para la neuroética porque en el marco predominante anglosajón en el que se plantea la teoría del proceso dual del juicio moral, la racionalidad moral es entendida en los términos utilitaristas del cálculo de consecuencias y en ocasiones se asimila al puro cálculo matemático ${ }^{11}$. Pero a poco que sondeemos la historia de la ética nos damos cuenta de que este planteamiento es excesivamente simplista y deformador. La racionalidad práctica no sólo no se reduce al cálculo de consecuencias (como sugiere Greene) sino que está directamente vinculada con las emociones de modo no reductivo. Es decir, el ejercicio del dar razón moral implica una serie de aspectos implicados en la naturaleza del ser humano, que son emocionales, pero también claramente reflexivos y discursivos. Pensar que ambos se encuentran disociados es falaz.

Una racionalidad a la altura de las investigaciones neurocientíficas no puede pretender estar disociada de sus sentimientos. Lo cual no quiere decir que los sentimientos sean previos y que la racionalidad no haga otra cosa más que justificar posteriormente una decisión ya tomada por las emociones morales ${ }^{12}$. Esto me parece central porque pensar que los sentimientos no están estrechamente vinculados con la conciencia reflexiva o la deliberación es tanto como eliminar el componente "hermenéutico» de la comprensión de sí de los propios sentimientos morales. ¿cómo podría llevarse a cabo dicha apropiación si no es a la luz de una autocomprensión ética personal de la propia vida y del propio cuerpo? Los juicios morales lo son de un sujeto moral cuyas decisiones están enraizadas en su organismo y en su historia vital como persona. El sujeto moral en tanto que autor se identifica con su propio cuerpo, dado que el cuerpo (Körper) es, en cada caso singular, el propio cuerpo $(\text { Leib })^{13}$. Siguiendo a Xavier Zubiri podemos decir que «el sentido de la corporeidad es radicalmente distinto de la corporeidad física ${ }^{14}$, hablamos ahora de «mi cuerpo», que encuentra su base en la «interioridad personal» ${ }^{15}$.

Por ello, el sujeto que se sabe en su ser corporal lo hace porque va ganando conciencia reflexiva. Dicha conciencia de la persona irremplazable físicamente se completa en el curso de su historia vital en la singularidad del individuo. El ser corporal queda in-corporado dentro de una perspectiva más amplia merced a «la voluntad ética anticipadora [que] proyecta la autocomprensión individual» ${ }^{16}$. Se trata por lo

11 Vid. Paxton, J.M., Bruni, T., Greene, J.D., «Are 'counter-intuitive' deontological judgments really counter-intuitive? An empirical reply to Kahane et al. (2012)», en: Social Cognitive and Affective Neuroscience, 9 (9), 2014 pp. 1368-1371.

12 Cortina, A., Neuroética y neuropolítica. Sugerencias para la educación moral, Tecnos, Madrid, 2011, p. 106.

13 «[S]e hace valer el sustrato orgánico de un cuerpo (Körper) que poseo como mi cuerpo sólo porque lo soy como cuerpo vivido (Leib)», HaBERmas, J., Entre naturalismo y religión, Alianza, Madrid, 2006, p. 195.

14 Zubiri, X., Sobre el problema de la filosofía y otros escritos (1932-1944), Alianza, Madrid, 2002, p. 262.

15 Vid. Conill, J., «La realidad personal en perspectiva neurocientífica. La aportación zubiriana», en: Pensamiento, vol. 71, núm. 266, 2015, pp. 253-271

16 Habermas, J., Entre naturalismo y religión, Alianza, Madrid, 2006, p. 196. 
tanto de la forja del carácter propio de una ética de fines y no sólo ni simplemente de una ética de móviles, o dicho de otra manera, que el elemento integrador no es el mero sentimiento sino el proyecto vital en el que se inscribe determinada conducta. Los juicios deontológicos como aplicación de principios morales universalizables se integran como un momento imprescindible en el marco de este proyecto vital que se sabe en el horizonte normativo de la moral.

Universidad de Valencia

Javier Gracia Calandín

javier.gracia@uv.es

[Artículo aprobado para publicación en diciembre de 2016] 